\title{
Utilización de Microsoft EXCEL en la enseñanza de sistemas eléctricos de potencia: desarrollo de un método matricial para la resolución del problema de despacho económico
}

\section{Manuel Alcázar-Ortega ${ }^{a}$, Carlos Álvarez-Bel ${ }^{a}$}

aDepartamento de Ingeniería Eléctrica, Universidad Politécnica de Valencia. Camino de Vera, s/n, edificio 5E, $2^{\circ}$ Planta. 46022 Valencia (España).

\begin{abstract}
The realization of lab practices in electrical power system requires the use of simulation programs, given the impossibility of performing field practices in many cases since experimenting with real electrical systems is unfeasible in many cases. However, the use of this kind of programs requires the purchase of expensive licenses and the specific learning of the use of each of them by students. This article makes evident the suitability of programs like Microsoft EXCEL, easily accessible and familiar to students, for the development of practices related to electrical power systems. In particular, a method for the systematic resolution of the economic dispatch problem is illustrated by means of a matrix method that can be easily implemented in a spreadsheet.
\end{abstract}

Keywords: economic dispatch, matrix method, electric power systems

\begin{abstract}
Resumen
La realización de prácticas de sistemas eléctricos de potencia requiere de la utilización de programas de simulación, dada la imposibilidad de realizar en muchos casos prácticas de campo por la inviabilidad de experimentar con sistemas eléctricos reales. Sin embargo, la utilización de dichos programas requiere la compra de costosas licencias y el aprendizaje específico de la utilización de cada uno de ellos por parte del alumno. Este artículo evidencia la idoneidad de programas como Microsoft EXCEL, fácilmente accesibles y con los que los alumnos están familiarizados, para la realización de prácticas relacionadas con los sistemas eléctricos de potencia. En particular, se ilustra un método para la resolución sistemática del problema de despacho económico utilizando un método matricial de resolución de ecuaciones lineales que puede ser fácilmente implementado en una hoja de cálculo.
\end{abstract}

Palabras clave: despacho económico, método matricial, sistemas eléctricos de potencia 


\section{Introducción}

Las prácticas de laboratorio son una de las mejores estrategias de aprendizaje en ingeniería (Montes Granada, 2004), utilizadas para afianzar los conocimientos adquiridos en clase y materializar, desde un punto de vista práctico, su aplicación a la vida profesional, consiguiendo que los estudiantes "aprendan haciendo" (Patiño, 2001). Sin embargo, en el caso de algunas áreas como los sistemas eléctricos de potencia, resulta inviable la realización de prácticas de campo dada la complejidad de dichos sistemas y la imposibilidad de someterlos a solicitaciones reales. Es por esto que, habitualmente, las prácticas de laboratorio en esta área están basadas en la realización de simulaciones mediante las que puede analizarse el comportamiento de sistemas, más o menos complejos, en los que se operan las redes y todos sus elementos tanto en régimen permanente como en régimen transitorio.

Existen diversos programas comerciales de simulación en base a los cuales se estructuran las prácticas que, en esta materia, se realizan en diversas universidades alrededor del mundo. Se pueden encontrar algunos ejemplos de manuales docentes de prácticas de sistemas eléctricos de potencia basados en programas de simulación genéricos como MATLAB (Balaji, 2010) o más específicos como PowerWorld (Glover et al., 2008), ETAP (Sridevi, 2015) o PSCADEMTDC (Mohan, 2010). Sin embargo, en este artículo se aborda la posibilidad de utilizar el paquete de Microsoft Office y, en particular, el programa Microsoft EXCEL, para la resolución de este tipo de problemas. De forma más detallada, la utilización de Microsoft EXCEL presenta las siguientes ventajas:

- Es una herramienta con la que los estudiantes están familiarizados, ya que se utiliza con frecuencia en múltiples ámbitos académicos y no académicos. Sin embargo, es cierto que la mayor parte de los usuarios no suele emplear toda la potencialidad que ofrece la herramienta para este tipo de análisis (números complejos, cálculo matricial), lo cual se favorece mediante la aplicación aquí propuesta.

- Es fácilmente accesible al estar instalada de serie en prácticamente la totalidad de los ordenadores personales. En cualquier caso, las universidades y otras instituciones académicas facilitan licencias en sus propios campus o para la utilización de los ordenadores personales de los alumnos ${ }^{1}$.

- A diferencia de los programas comerciales de simulación, donde el alumno se limita a introducir unos parámetros y a interpretar los resultados obtenidos, la utilización de Microsoft EXCEL requiere que sea el propio alumno quien cree el programa de cálculo mediante la introducción de las ecuaciones desarrolladas a partir de los conocimientos teóricos adquiridos en clase que, de esta forma, se aplican directamente.

- En el caso de procedimientos iterativos, como los requeridos para la resolución del problema de flujo de carga, estimación del estado o el despacho económico con pérdidas en la red, Microsoft EXCEL muestra el resultado tabulado de cada una de las iteraciones realizadas en una hoja de cálculo, lo que facilita la detección de

\footnotetext{
${ }^{1}$ Por ejemplo, la Universidad Politécnica de Valencia proporciona acceso a la licencia educativa de Microsoft Office 365 que tiene contratada para sus estudiantes y personal.
}

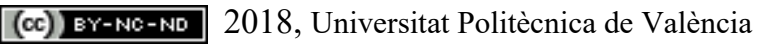


errores por parte del propio alumno. De esta forma, el estudiante aprende de sus propios errores, lo que afianza el proceso de aprendizaje (Sasso, 2015).

Los autores han venido proponiendo diversas aplicaciones prácticas en el campo de los sistemas eléctricos de potencia (Álvarez Bel y Alcázar Ortega, 2012) de cara a su resolución con Microsoft EXCEL. En particular, este artículo presenta un método de cálculo para abordar el problema del despacho económico en sistemas eléctricos mediante cálculo matricial que puede ser realizado fácilmente con la ayuda de una hoja de cálculo. La resolución sistemática de este tipo de problemas resulta básica de cara a afrontar casos más complejos (por ejemplo, para la programación de unidades de generación en sistemas multiárea) donde es necesario solucionar diferentes despachos económicos de forma iterativa hasta alcanzar la solución al problema planteado (Wook y Wollenberg, 1984).

El artículo se estructura de la siguiente forma: El capítulo 2 presenta los objetivos de la aplicación descrita en este documento, los cuales se desarrollarán de forma detallada en el capítulo 3, incluyendo su fundamento teórico y su implementación mediante el método matricial. El capítulo 4 muestra los resultados obtenidos en un caso práctico donde el método matricial se ha implementado mediante Microsoft EXCEL. Finalmente, las conclusiones del presente trabajo se incluyen en el capítulo 5 .

\section{Objetivos}

Los objetivos del trabajo que aquí se presenta son los siguientes:

- En primer lugar, presentar las ventajas que ofrece Microsoft EXCEL para el análisis de problemas prácticos relacionados con el estudio de los sistemas eléctricos de potencia

- Describir un método desarrollado por los autores para resolver, de forma sistemática, problemas prácticos de despacho económico en sistemas eléctricos de potencia, así como su implementación en una hoja de cálculo

- Mostrar a los docentes de este campo las potencialidades que la utilización de Microsoft EXCEL presenta para la realización de prácticas de sistemas eléctricos de potencia frente a otros programas de simulación utilizados habitualmente.

- Finalmente, ilustrar a los alumnos sobre cómo resolver problemas prácticos en el área de los sistemas eléctricos con una hoja de cálculo.

\section{Desarrollo de la innovación}

\subsection{Fundamento teórico}

El despacho económico es una estrategia utilizada en el análisis y operación de los sistemas eléctricos de potencia para determinar la cantidad de potencia activa que debe ser entregada por cada una de las unidades de generación conectadas al sistema para satisfacer la demanda de los consumidores al menor coste posible. Es, por tanto, una herramienta económica que se basa en optimizar los costes de producción de cada uno de los generadores conectados al 
Utilización de Microsoft EXCEL en la enseñanza de sistemas eléctricos de potencia: desarrollo de un método matricial para la resolución del problema de despacho económico

sistema, siendo necesario un modelo económico de cada unidad de producción que represente tanto sus costes fijos como variables (coste del combustible, costes de operación, costes de mantenimiento, etcétera).

El coste individual de producción de una unidad de generación $i$ suele representarse mediante una ecuación polinómica de segundo o tercer grado (Glover et al., 2008) de acuerdo con la siguiente expresión:

$$
C_{i}=a_{i}+b_{i} \cdot P_{G_{i}}+c_{i} \cdot P_{G_{i}}^{2}+d_{i} \cdot P_{G_{i}}^{3} € / h
$$

donde:

- $\quad P_{G i}$ es la potencia activa horaria entregada al sistema por la unidad ${ }_{\mathrm{i}}$ expresada en MW

- $a_{i}$ representa el coste horario fijo de producción de la unidad $i$, en $€ / \mathrm{h}$

- $b_{i}, c_{i} \mathrm{y} d_{i}$ representa el coste variable de producción, en función de la potencia producida, de la unidad $i$, en $€ / \mathrm{MWh}$

El despacho económico es un problema de optimización, donde se determina el valor de las potencias generadas por cada unidad de generación conectada al sistema al menor coste posible. No obstante, el valor de dichas potencias no puede ser arbitrario y está sujeto a las siguientes restricciones de igualdad y desigualdad:

- Restricción de igualdad: la potencia total entregada por los $N$ generadores del sistema debe ser igual a la potencia total demandada por los consumidores más las pérdidas producidas en las redes de transporte y distribución:

$$
\sum_{i=1}^{N} P_{G_{i}}=P_{D}+P_{\text {pérdidas }} \quad M W
$$

- Restricciones de desigualdad: Dado que los generadores no pueden trabajar fuera de sus márgenes de operación, la potencia generada por cada uno de ellos estará acotada por sus valores máximo y mínimo de producción:

$$
P_{G_{i}}^{\min } \leq P_{G_{i}} \leq P_{G_{i}}^{\operatorname{máx}} \quad M W
$$

Para el caso del método propuesto en este trabajo, se considerará que no existen pérdidas de potencia en los diferentes elementos del sistema, lo cual, aunque inadmisible desde el punto de vista real (las pérdidas pueden alcanzar el 15\% de la potencia total generada), resulta de 
especial utilidad para comprender el problema. Si se desprecian las pérdidas, la restricción de igualdad queda de la siguiente manera:

$$
\sum_{i=1}^{N} P_{G_{i}}=P_{D} \quad M W
$$

El procedimiento para llevar a cabo la optimización es el siguiente (Elgerd, 1982):

- Paso 1: Se calcula el valor de potencia producido por cada generador para minimizar el coste total de producción, teniendo en cuenta la restricción de igualdad. Para ello, se construye una función objetivo $F$ que incluye el coste total de producción y la restricción de igualdad multiplicada por el operador de Lagrange $\lambda$ :

$$
F\left(P_{G_{1}}, P_{G_{2}}, \ldots P_{G_{N}}, \lambda\right)=\sum_{i=1}^{N} C_{i}+\lambda\left(P_{D}-\sum_{i=1}^{N} P_{G_{i}}\right) \quad M W
$$

- Paso 2: Se calculan las derivadas parciales de la función objetivo y se igualan a cero para obtener un sistema de ecuaciones que permita determinar la potencia entregada por cada unidad de generación con un coste total mínimo:

$$
\left\{\begin{array}{l}
\frac{\partial F}{\partial P_{G_{1}}}=\frac{\partial C_{1}}{\partial P_{G_{1}}}-\lambda=0 \\
\frac{\partial F}{\partial P_{G_{2}}}=\frac{\partial C_{2}}{\partial P_{G_{2}}}-\lambda=0 \\
\vdots \\
\sum_{i=1}^{N} P_{G_{i}}=P_{D}
\end{array}\right.
$$

- $\quad$ Paso 3: Del cálculo anterior resulta un sistema de $(\mathrm{N}+1)$ ecuaciones con $(\mathrm{N}+1)$ incógnitas, el cual se puede resolver a través de diversos métodos de cálculo. El resultado son las $N$ potencias entregadas por cada uno de los $N$ generadores, así como el valor de $\lambda$. El parámetro $\lambda$ representa el coste incremental de operación del área y constituye el coste de producir una unidad adicional de potencia.

(c)) EY-NC-ND 2018, Universitat Politècnica de València 
- Paso 4: Obtenidos los valores de potencia entregada por cada generador, deben verificarse las restricciones de desigualdad, de forma que los generadores cuya potencia quede fuera de sus límites técnicos será fijado a su máximo (o mínimo) técnico y se recalculará la optimización con las unidades restantes.

\subsection{Desarrollo del método matricial}

El sistema de ecuaciones mostrado en (6) puede resolverse utilizando diversos métodos. El método matricial aquí desarrollado tiene las siguientes ventajas:

- Se construye fácilmente a partir de los coeficientes de las curvas de coste de los generadores participantes en el despacho, por lo que no es necesario desarrollar el sistema de ecuaciones en cada caso.

- Se resuelve fácilmente en dos pasos, siendo su única complejidad la inversión de una matriz y su producto por un vector columna.

- Es fácilmente programable, lo que facilita su utilización en sistemas con un gran número de generadores. En particular, en la sección siguiente se explicará cómo dicho método puede aplicarse para la resolución del despacho económico mediante Microsoft EXCEL.

Si se sustituyen las curvas de coste de los generadores en (6), construidas tal y como se indica en (1), el sistema de ecuaciones resultante puede escribirse de la siguiente manera:

$$
\left\{\begin{array}{l}
b_{1}+2 \cdot c_{1} \cdot P_{G_{1}}-\lambda=0 \\
b_{2}+2 \cdot c_{2} \cdot P_{G_{2}}-\lambda=0 \\
\vdots \\
b_{N}+2 \cdot c_{N} \cdot P_{G_{N}}-\lambda=0 \\
P_{G_{1}}+P_{G_{1}}+\ldots+P_{G_{N}}=P_{D}
\end{array}\right.
$$

Reordenando (7) para dejar en el primer miembro de cada ecuación los términos variables y en el segundo miembro los términos independientes, el sistema quedaría como sigue.

$$
\left\{\begin{array}{l}
2 \cdot c_{1} \cdot P_{G_{1}}-\lambda=-b_{1} \\
2 \cdot c_{2} \cdot P_{G_{2}}-\lambda=-b_{2} \\
\vdots \\
2 \cdot c_{N} \cdot P_{G_{N}}-\lambda=-b_{N} \\
P_{G_{1}}+P_{G_{1}}+\ldots+P_{G_{N}}=P_{D}
\end{array}\right.
$$


En forma matricial, dicho sistema se podría escribir de la siguiente forma:

$$
\left[\begin{array}{ccccc}
2 \cdot c_{1} & 0 & 0 & 0 & -1 \\
0 & 2 \cdot c_{2} & 0 & 0 & -1 \\
\vdots & \vdots & \ddots & \vdots & \vdots \\
0 & 0 & 0 & 2 \cdot c_{N} & -1 \\
1 & 1 & 1 & \cdots & 0
\end{array}\right] \times\left[\begin{array}{c}
P_{G_{1}} \\
P_{G_{2}} \\
\vdots \\
P_{G_{N}} \\
\lambda
\end{array}\right]=\left[\begin{array}{c}
-b_{1} \\
-b_{2} \\
\vdots \\
-b_{N} \\
P_{D}
\end{array}\right]
$$

obteniéndose un sistema de la forma: $[\mathrm{A}] \mathrm{x}[\mathrm{P}]=[\mathrm{B}]$. El despacho económico consistirá, por tanto, en obtener los valores del vector columna $[\mathrm{P}]$ a partir de los valores de la matriz $[\mathrm{A}] \mathrm{y}$ del vector columna [B].

Analizando en detalle las características de las matrices [A] y [B], se puede concluir lo siguiente:

- La matriz [A] es una matriz cuadrada de dimensiones $(\mathrm{N}+1) \mathrm{x}(\mathrm{N}+1)$, siendo $N$ el número de generadores participantes en el despacho. Esta matriz puede descomponerse en cuatro submatrices de acuerdo con la siguiente estructura:

$$
[A]=\left[\begin{array}{l|l}
A_{1} & A_{2} \\
\hline A_{3} & A_{4}
\end{array}\right]
$$

La submatriz $\mathrm{A}_{1}$ es una matriz diagonal de dimensiones NxN. Si se asocia cada fila $i$ y columna $i$ con el generador $i$, el elemento de la diagonal principal $\mathrm{A}_{1-\mathrm{i}, \mathrm{i}}$ será igual al coeficiente que multiplica a $P_{G i}{ }^{2}$ en la curva de costes del generador $i$ multiplicado por dos.

La submatriz $\mathrm{A}_{2}$ es un vector columna de dimensión $\mathrm{Nx} 1$ en el que todos los elementos son igual a -1 .

La submatriz $A_{3}$ es un vector fila de dimensión $1 \times N$ en el que todos sus elementos son igual a 1 .

Finalmente, la submatriz $\mathrm{A}_{4}$ tiene dimensión $1 \mathrm{x} 1 \mathrm{y}$ es igual a cero.

- $\quad$ El vector columna [B] tiene dimensiones $(\mathrm{N}+1) x 1$ y sus $N$ primeros elementos para cada fila $i$ son iguales al coeficiente que multiplica a $P_{G i}$ en la curva de costes del generador $i$. El último elemento de la columna es igual a la demanda total del sistema, la cual debe ser satisfecha por todos los generadores participantes en el despacho.

El vector columna $[\mathrm{P}]$ contiene todas las incógnitas que deben ser calculadas en el despacho económico, a saber: la potencia entregada por cada uno de los generadores y el valor del coste incremental de operación del área $\lambda$.

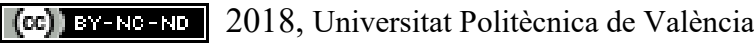


Construidas las matrices $[\mathrm{A}] \mathrm{y}[\mathrm{B}]$ a partir de las curvas de costes de todos los generadores y el valor de la potencia total demandada en el sistema, $P_{D}$, el vector de incógnitas puede calcularse a partir de la siguiente expresión:

$$
[P]=\left[\begin{array}{c}
P_{G_{1}} \\
P_{G_{2}} \\
\vdots \\
P_{G_{N}} \\
\lambda
\end{array}\right]=[A]^{-1} \times[B]
$$

Una vez calculadas todas las incógnitas, es necesario verificar las restricciones de desigualdad. Se pueden dar los siguientes casos:

- Existen generadores en los que $P_{G_{i}}>P_{G_{i}}^{m a x}$. Si algún generador supera en la solución del despacho su potencia máxima, se fijará a su producción a dicho valor máximo y será retirado del despacho. Ello se traduce en la eliminación de la fila y columna correspondientes a dicho generador en las matrices $[\mathrm{A}],[\mathrm{B}]$ y $[\mathrm{P}]$ y en la modificación de la potencia total demandada a cubrir por los generadores en despacho, a la que habrá que sustraer la que se ha asignado al generador retirado.

- Existen generadores en los que $P_{G_{i}}<P_{G_{i}}^{\min }$. Estos generadores serán mantenidos al realizar una nueva evaluación del despacho económico después de retirar aquellos que hubieran superado su potencia máxima ya que, en un nuevo redespacho, podría serles asignada parte de la potencia excedente de los generadores retirados $\mathrm{y}$, con ello, pasarían a operar por encima de su valor mínimo.

- Los generadores que satisfagan las restricciones de desigualdad serán aquellos que, cubriendo la demanda total asignada al menor coste posible, son físicamente capaces de poder hacerlo.

\section{Resultados: resolución mediante la utilización de Microsoft EXCEL}

En este apartado se presenta la aplicación del método propuesto anteriormente a un caso práctico, que se resolverá mediante la utilización de Microsoft EXCEL. Considérense cuatro sistemas eléctricos interconectados, tal y como se muestra en la Figura 1, cada uno de los cuales dispone de recursos propios de generación que deben satisfacer una demanda conjunta de $1.500 \mathrm{MW}$.

Cada una de las cuatro áreas tiene unos costes de generación que se pueden estimar a partir de las siguientes curvas:

$$
\begin{aligned}
& C_{1}=280+13,2 \cdot P_{G_{1}}+0,08 \cdot P_{G_{1}}{ }^{2} \quad \epsilon / h \\
& C_{2}=315+10,5 \cdot P_{G_{2}}+0,06 \cdot P_{G_{2}}{ }^{2} \quad \epsilon / h
\end{aligned}
$$




$$
\begin{aligned}
& C_{3}=187+11,7 \cdot P_{G_{3}}+0,09 \cdot P_{G_{3}}{ }^{2} \quad \epsilon / h \\
& C_{4}=255+9,2 \cdot P_{G_{4}}+0,10 \cdot P_{G_{4}}{ }^{2} \quad \epsilon / h
\end{aligned}
$$

Las potencias máximas y mínimas que pueden ser producidas por cada generador son las siguientes:

$$
\begin{aligned}
& 50 \leq P_{G_{1}} \leq 550 \quad M W \\
& 75 \leq P_{G_{2}} \leq 750 \quad M W \\
& 20 \leq P_{G_{3}} \leq 400 \quad M W \\
& 10 \leq P_{G_{4}} \leq 250 \quad M W
\end{aligned}
$$

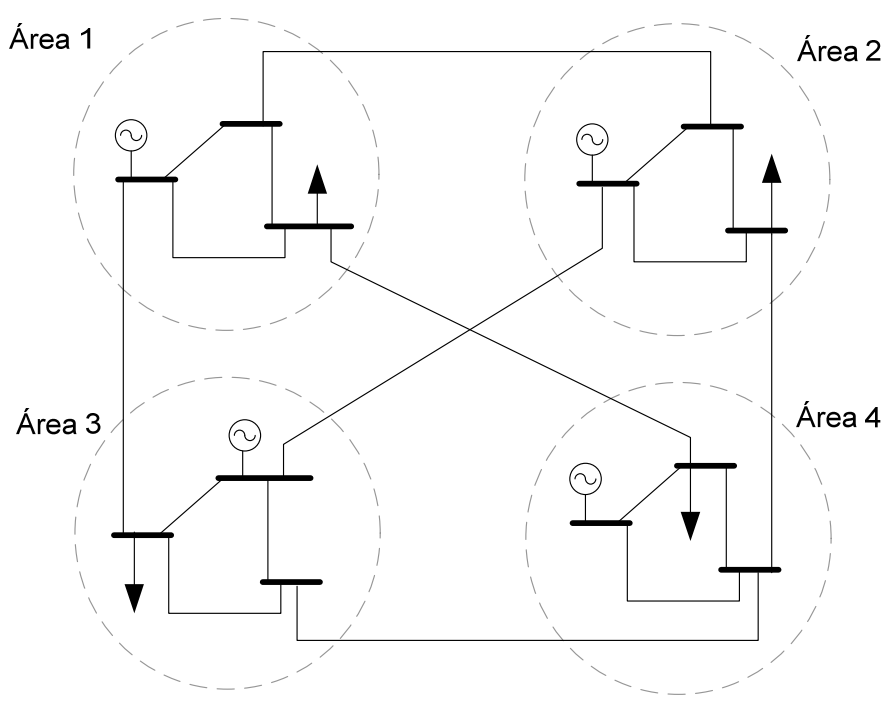

Figura 1. Caso de aplicación con cuatro áreas eléctricas interconectadas

De acuerdo con la metodología planteada en la sección anterior, los coeficientes que se debería utilizar para la construcción de las matrices $[\mathrm{A}]$ y $[\mathrm{B}]$ procedentes de las expresiones anteriores serían los siguientes: 


$$
[A]=\left[\begin{array}{ccccc}
2 \cdot c_{1} & 0 & 0 & 0 & -1 \\
0 & 2 \cdot c_{2} & 0 & 0 & -1 \\
0 & 0 & 2 \cdot c_{3} & 0 & -1 \\
0 & 0 & 0 & 2 \cdot c_{4} & -1 \\
1 & 1 & 1 & 1 & 0
\end{array}\right]=\left[\begin{array}{ccccc}
0,16 & 0 & 0 & 0 & -1 \\
0 & 0,12 & 0 & 0 & -1 \\
0 & 0 & 0,18 & 0 & -1 \\
0 & 0 & 0 & 0,20 & -1 \\
1 & 1 & 1 & 1 & 0
\end{array}\right]
$$

$$
[B]=\left[\begin{array}{c}
-b_{1} \\
-b_{2} \\
-b_{3} \\
-b_{4} \\
P_{D}
\end{array}\right]=\left[\begin{array}{c}
13,2 \\
-10,5 \\
-11,7 \\
-9,2 \\
1500
\end{array}\right]
$$

La solución del despacho económico se obtendría calculando el vector columna [P] a partir de la expresión (11). Para hacerlo con Microsoft EXCEL habrá que seguir los siguientes pasos:

i) Escribir en un bloque de celdas $5 \times 5$ los coeficientes de la matriz [A], y en otro bloque de celdas $5 \times 1$ los coeficientes de la matriz [B], de acuerdo con las expresiones (20) y (21)

ii) Calcular la matriz $[\mathrm{A}]^{-1}$. Para ello:

a. Seleccionar en la hoja de cálculo una región $5 \times 5$ donde la matriz inversa debe estar escrita.

b. Escribir en la celda superior izquierda de dicha región la expresión:

$$
=\operatorname{MINVERSA}((1,1):(5,5))
$$

Siendo $(1,1)$ la celda superior izquierda de la región donde aparecerá la matriz inversa, y $(5,5)$ la celda inferior derecha. En la Figura 2, donde se muestra la resolución completa del ejemplo con Microsoft EXCEL, la referencia $(1,1)$ correspondería con la celda $\mathrm{C} 3$, mientras que $(5,5)$ sería la celda G7.

c. Pulsar la tecla ENTER. Al hacerlo, aparecerá en la celda $(1,1)$ el elemento $(1,1)$ de la matriz inversa.

d. Con el ratón (o con las teclas de desplazamiento), seleccionar toda la región de la hoja de cálculo donde debe aparecer la matriz inversa. Debe ser un bloque $5 \times 5$.

e. Pulsar la tecla F2 para que la expresión (22) adquiera carácter editable y, a continuación, pulsar simultáneamente las tecla Ctrl, Alt y ENTER. Al 
hacerlo, aparecerá la matriz inversa $[\mathrm{A}]^{-1}$ completa, tal y como se muestra en la Figura 2.

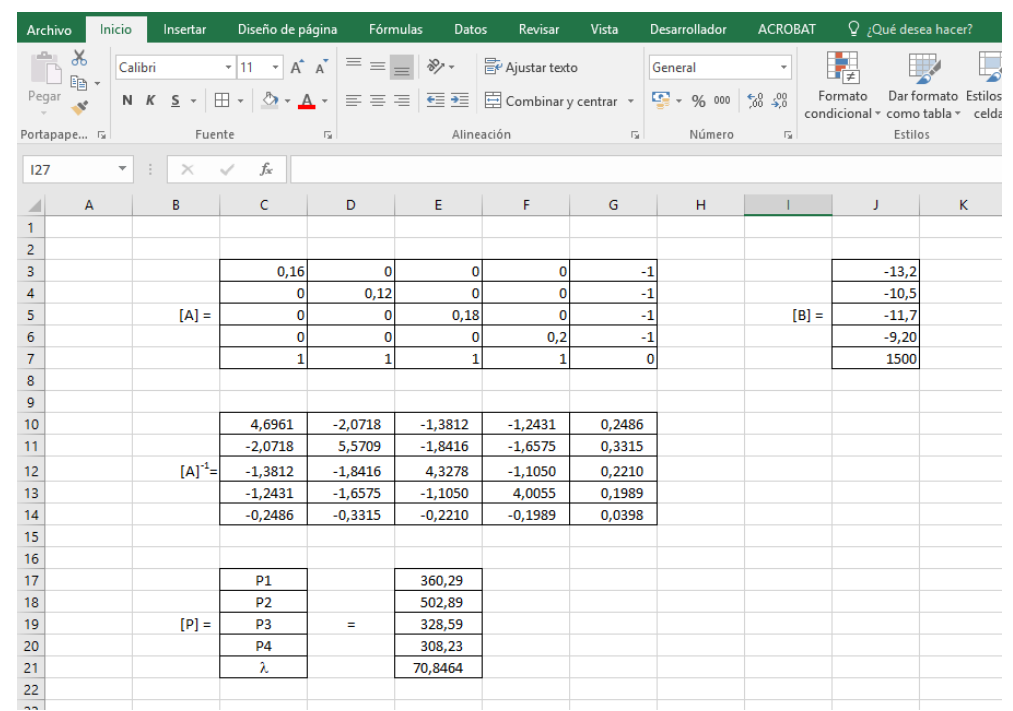

Figura 2. Resolución del sistema matricial mediante Microsoft EXCEL

iii) Calcular $[\mathrm{P}]$ como producto de la matriz $[\mathrm{A}]^{-1}$ y la matriz [B]. Para ello se seguirá un procedimiento similar al del cálculo de la matriz inversa:

a. Seleccionar en la hoja de cálculo la región $5 \times 1$ donde aparecerán los coeficientes del vector columna [P].

b. Escribir en la celda superior izquierda de dicha región la expresión:

$=M M U L T\left(\right.$ rango de celdas de $[A]^{-1}$; rango de celdas de $\left.[B]\right)$

En el ejemplo mostrado en la Figura 2, dicha expresión sería la siguiente:

$$
=M M U L T(C 10: G 14 ; J 3: J 7)
$$

c. Pulsar la tecla ENTER. Al hacerlo, aparecerá en la celda superior del vector $[\mathrm{P}]$ el elemento $(1,1)$ de dicho vector.

d. Con el ratón (o con las teclas de desplazamiento), seleccionar toda la región de la hoja de cálculo donde debe aparecer el vector [P]. Debe ser un bloque $5 \times 1$.

e. Pulsar la tecla F2 para que la expresión (23) adquiera carácter editable y, a continuación, pulsar simultáneamente las tecla Ctrl, Alt y ENTER. Al hacerlo, aparecerá el vector columna $[\mathrm{P}]$.

De acuerdo con la resolución al ejercicio planteado, los valores de la potencia entregada por cada área serían de 360,29 MW para el área 1, 502,89 MW para el área 2, 328,59 MW para el área 3 y 308,23 MW para el área 4. De acuerdo con las expresiones (15), (16), (17) y (18), 
Utilización de Microsoft EXCEL en la enseñanza de sistemas eléctricos de potencia: desarrollo de un método matricial para la resolución del problema de despacho económico

los generadores de las tres primeras áreas tienen una producción adecuada, ya que se encuentran entre sus respectivos límites superior e inferior. En cambio, el área 4 tiene una potencia máxima de $300 \mathrm{MW}$ mientras que el despacho le ha asignado una cantidad superior. Para solucionar esta restricción, tal y como se indicó en la metodología, hay que fijar la potencia del área 4 en su límite superior de 300 MW y recalcular el despacho entre los generadores de las áreas restantes. Al utilizar el método matricial, este cálculo es inmediato, ya que sólo hay que eliminar la fila y la columna correspondiente al generador 4 de las matrices $[\mathrm{A}]$ y $[\mathrm{B}]$ y volver a calcular el resultado del vector columna $[\mathrm{P}]$, donde también se ha eliminado la fila correspondiente al área sustraída del despacho.

El resultado se muestra en la Figura 3, donde el sistema obtenido se ha reducido en una dimensión ( $4 \mathrm{x} 4$ para la matriz [A]' y 4x1 para los vectores columna [B]' y [P]'). La fila y columna eliminadas aparecen sombreadas en color gris. Asimismo, se observa cómo la última fila del vector columna [B]' muestra la demanda total del sistema menos la que ya se ha fijado a la generación del área $4(1500-300 \mathrm{MW})$.

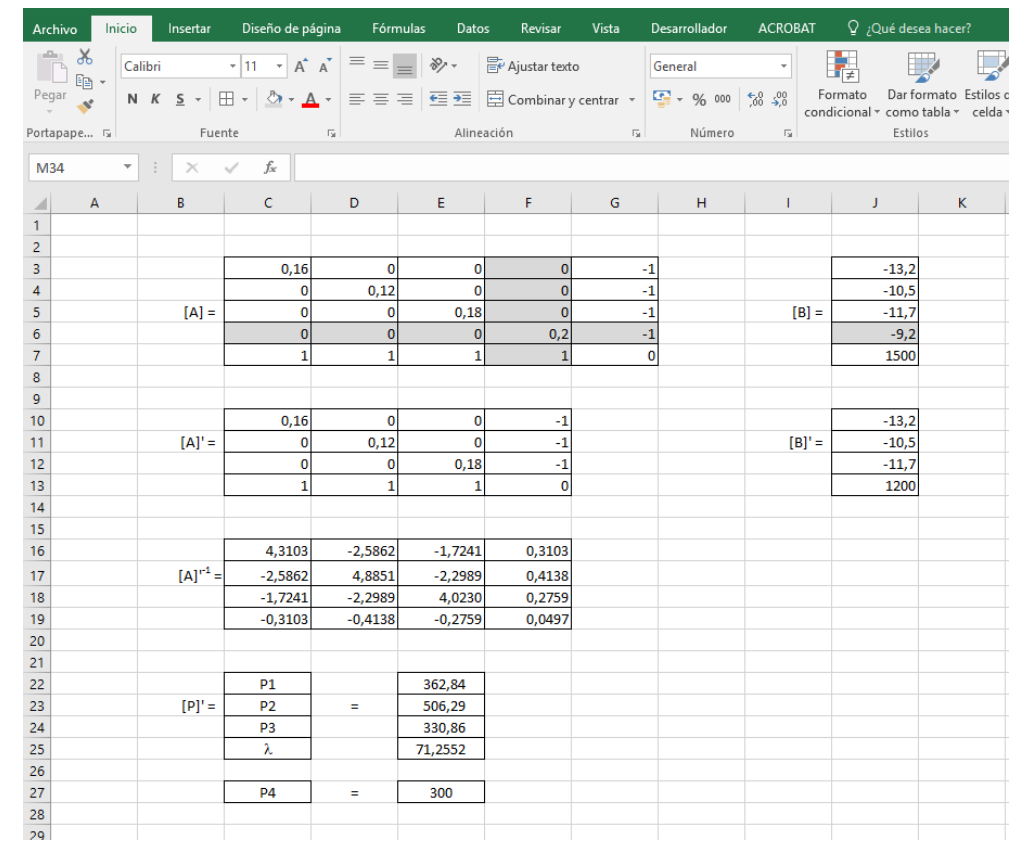

Figura 3. Resolución del sistema matricial mediante Microsoft EXCEL. Eliminación de la restricción del área 4

En este caso, todas las potencias del despacho están acotadas por los límite superior e inferior de cada área, por lo que ésta sería la solución definitiva del despacho. 


\section{Conclusiones}

Este artículo presenta un método matricial para la resolución del problema de despacho económico sin pérdidas en sistemas eléctricos de potencia, mediante el que es posible obtener soluciones rápidas con la sola utilización de los coeficientes de la función de costes de los generadores participantes en el despacho.

Una de las principales ventajas de este método es la posibilidad de agregar o eliminar cualquier generador o área productora, simplemente añadiendo o eliminando una fila y columna en las matrices [A] y [B], así como la facilidad de repetir este cálculo tantas veces como sea necesario de cara a verificar el cumplimiento de las restricciones de los generadores participantes.

Asimismo, se ilustra la aplicación de dicho método para la resolución sistemática del problema de despacho económico en Microsoft EXCEL, que puede ser fácilmente implementado en una hoja de cálculo.

Este software presenta múltiples ventajas, tales como su accesibilidad y popularidad entre los estudiantes, su facilitad de utilización o su idoneidad para la resolución de procedimientos iterativos. Además, desde el punto de vista educacional, Microsoft EXCEL resulta idóneo para trabajar desde la base la resolución de los problemas prácticos relacionados con las clases teóricas. Ello es debido a que, a diferencia de los programas comerciales donde el estudiante introduce unos parámetros y obtiene directamente unos resultados, aquí se requiere que sea el pripio alumno quien cree el programa de cálculo mediante la introducción de las ecuaciones básicas que rigen el problema analizado. Ello hace de Microsoft EXCEL una herramienta de excepcional utilidad que, aunque es poco utilizada en el área de los sistemas eléctricos de potencia, resulta muy versátil en la realización de ejercicios prácticos aplicados a este campo.

\section{Referencias}

ÁlVAREZ BEL, C. y ALCÁZAR ORTEGA, M. (2012). Prácticas de Sistemas Eléctricos de Potencia. Universitat Politècnica de València. Ref. 0461P03

BALAJI, V. (2010). Power system simulation laboratory. Dhanalakshmi College of Engineering. DOI: 10.13140/RG.2.1.4794.6485

ELGERD, O. (1982). Electric energy systems theory: an introduction. McGraw-Hill, New York, USA, ISBN 0070192308

GLOVER, J., SARMA, M.S., OVERBYE, T. (2008). Power systems. Analysis and design. Thompson Corporation, Toronto, Canada. ISBN 9780534548841

MOHAN, N. (2010). Power Systems Laboratory. University of Minnesota, Minneapolis, MN, USA. 
Utilización de Microsoft EXCEL en la enseñanza de sistemas eléctricos de potencia: desarrollo de un método matricial para la resolución del problema de despacho económico

MONTES GRANADA, W.F. (2004). "Prácticas de laboratorio en ingeniería: una estrategia efectiva de aprendizaje" en Notas Universitarias, vol. 4, núm. 11. Ibagué, Colombia. CEDIP, ISSN: 1794-1997.

PATIÑO, G. (2001). "El taller, un continuum entre enseñanza y aprendizaje. La Práctica de la Enseñanza” en Notas Universitarias. Ibagué, Colombia. CEDIP, ISSN: 1794-1997

SASSO, P. (2015). "El error como herramienta del aprendizaje" en Reflexión Académica en Diseño y Comunicación, vol. 25, p. 25-27. Buenos Aires, Argentina, ISSN 1668-1673.

SRIDEVI, J. (2015). Power systems Lab. Gokaraju Rangaraju Institute of Engineering and Technology.

WOOK, A.J., WOLLENBERG, B.F. (1984). Power generation, operation and control. John Willey and Sons, New York, USA. ISBN 0471586994 